\title{
Grammar and Gesture in American Sign Language: Implications for Constructing Meaning
}

\author{
SCOTT K. LIDDELL \\ Gallaudet University
}

\section{Introduction}

Stokoe (1960) was the first to argue that American Sign Language (ASL) demonstrates the kind of abstract structure one expects to find in the analysis of any spoken language. ${ }^{1}$ The idea that ASL was not a real language was so deeply embedded in the culture, however, that it required nearly two decades for Stokoe's claim to gain acceptance. For Stokoe's idea to take hold, it was necessary to give up the idea that ASL was a poor gestural substitute for real language. Linguists analyzing ASL found morphemes where previously there had been only gestures. The transformation from gesture to morpheme was so complete that, in the end, no gestures remained.

The fact that some signs point toward things is undeniable. ASL pronouns, for example, point toward physically present referents. The pointing, however, has not become part of the analysis of these pointing signs. The field has come to accept that even when a referent is physically present, a sign is articulated at an area of space associated with the physically present referent. Claiming that signs are articulated with respect to locations in space avoids the necessity of claiming that signs actually point at things. The analysis is the same for non-present referents, where an area of space is also associated with the non-present referent. In either case, signs are seen as being directed toward morphemic areas of space.

Liddell (1995) argues that if the referent is physically present, signs are not directed toward areas of space at all, but rather, toward the referent. In addition, I analyze areas of space associated with non-present referents as conceptual entities rather than morphemes. Furthermore, signs point at such areas of space because of the conceptual ability to point. This challenges the notion that signs point because the signer is articulating a spatial morpheme. There has been considerable opposition to the idea that what looks like pointing really is pointing. Part of the reason

\footnotetext{
${ }^{1}$ I would like to thank MJ Bienvenu, Melissa Draganac, Paul Dudis, and Greg Visco for very useful discussions of the ASL data and for providing their native speaker intuitions about the use of directional verbs.
} 
Scott K. Liddell

for this is that it gives the appearance of a return to the pre-Stokoe idea that ASL was merely a collection of gestures rather than a language. A second reason for the opposition rests with assumptions within the field of linguistics itself. Ideas about the structure of ASL were being transformed during the seventies and eighties. The operating principles during those times held that the meaning of a sentence comes from its morphemic parts and their grammatical arrangement. Langacker (1991) refers to this as the "building block metaphor." Since directing signs toward things is clearly meaningful, there was no choice but to make morphemes responsible for both the meaning and the directionality.

\section{Directional Pronouns in ASL}

There is a significant articulatory difference between all sign languages and all spoken languages. In producing spoken words, the tongue does not point at things in the environment as a speaker produces words. In contrast to this apparently universal characteristic of speech, several classes of signs do point at things. That is, in order to properly produce many ASL signs, the hands must be directed toward real people or things being talked about, if those real people or things are present. All known sign languages include such signs.

During the past thirty years, there have been numerous proposed morphemic analyses of directional signs including Woodward (1970), Lacy (1974), Fischer (1975), Friedman (1975), Edge and Herrmann (1977), Klima and Bellugi (1979), Gee and Kegl (1982), Padden (1988), and Neidle et al. (1995). All such proposals are based on the idea that morphemic locations in the space ahead of the signer become associated with a referent-whether physically present or not. The analyses assume that incorporating a spatial locus into the structure of the verb causes the spatial locus to become the point of articulation for the verb. This causes the hand as articulator to move toward that locus.

Liddell $(1994,1995)$ demonstrates that the assumption upon which these analyses are based is false. That is, signs do not move toward a single spatial locus. Analyses based on the idea that signs do move this way are inconsistent with observable facts about how signs are actually produced. I have argued that pronouns and directional verbs either point at physically present referents themselves or point at conceptualized-as-present referents. I will not reiterate the arguments here, but rather, will take it as given that the types of directional signs discussed in this paper actually point at things. Sign types that can be gradiently placed or directed for the purpose of pointing include some nouns, almost all pronouns, a large set of verbs, numeral quantifiers in general, signs expressing locative meanings, and even signs for alphabetic characters.

Signs can point by the directionality of their movement. The way the hand is oriented can also point. For example, in Figure 1 the singular, non-first person pronoun PRO has the index finger directed toward and also moves toward the addressee. In Figure 1a, PRO is directed horizontally toward the addressee's chest. In Figure 1b, PRO is also directed toward the addressee's chest, but its form differs from the sign in Figure 1a. The reason for this is straightforward. Since the 
referent is standing in Figure 1b, PRO must be directed upward in order to point at his chest.

\section{Figure 1}

a. PRO

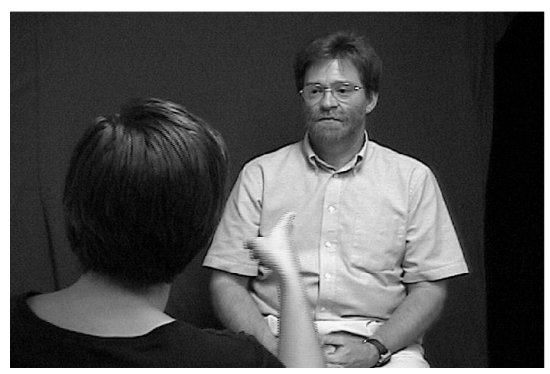

b. PRO

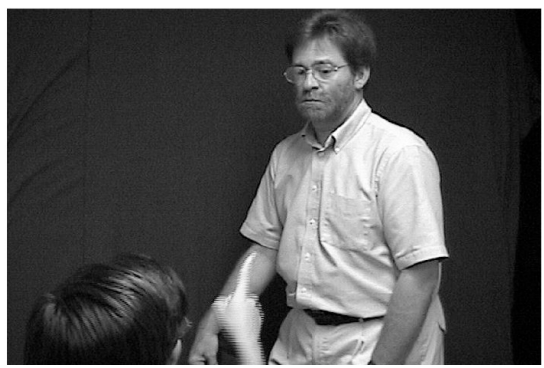

In Figure 2a, the dual first person pronoun makes an in-and-out rocking motion between the signer and the other person it is directed toward. The motion is primarily due to bending the wrist toward and then away from the signer. The inand-out motion produces a line whose two ends point at the two referents of the pronoun. Motion of the wrist also causes the non-first person dual pronoun in Figure $2 \mathrm{~b}$ to make a sideways back-and-forth movement that also points toward the pronoun's two referents.

\section{Figure 2}

a. PRO-1-DUAL

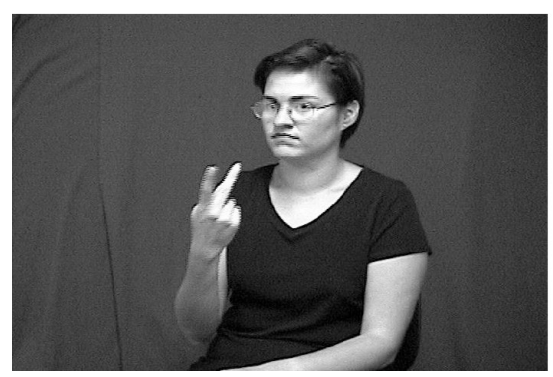

b. PRO-DUAL

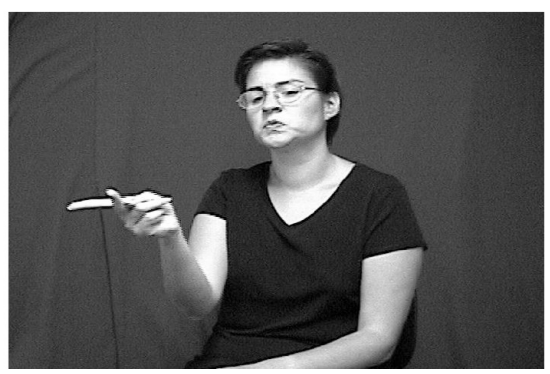

\section{Accounting for Directionality}

The signs in Figures 1 and 2 illustrate the general point that ASL pronouns have fixed lexical meanings and derive their contextually variable referential values from their directionality. The referential values are indicated by the directionality of the signs themselves. This fact about ASL is easily accommodated using concepts from cognitive grammar (Langacker 1987, 1991) and mental space theory (Fauconnier 1985, 1997).

In cognitive grammar a noun or pronoun profiles a thing in some domain. This constitutes the lexically encoded meaning of the noun or pronoun. Figure 3 contains two representations of the semantic structure of PRO. In Figure 3a, the semantic structure includes two entities: the signer, represented as a circle with an 
' $S$ ' inside it, and one additional entity. The second entity, represented as a bold circle, is the profiled entity in the semantic structure of PRO. This is a diagrammatic way of representing some entity other than the signer. An abbreviated representation of the same semantic structure that only represents the profiled entity itself appears in Figure 3b. I will use this type of abbreviated representation in subsequent diagrams.

\section{Figure 3}

a.

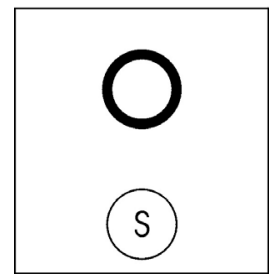

b.

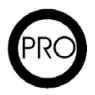

Liddell (1995) proposes that one's conception of the immediate environment is a mental space called real space. Real space differs from other mental spaces in that the entities within it are conceptualized as existing in the immediate environment itself. In general, our conceptualizations of immediate reality conform well to the actual physical elements we assume are responsible for our perceptions. That is, I take in perceptions, and construct real space based on those perceptions. If my real space includes a pencil on a desk in front of me, then I have confidence that if I extend my hand toward the real space pencil, I will make contact with a physical pencil. Instances where real space does not conform to the actual locations of physical entities help show the distinction between conceptual entities in real space and physical things. For example, Figure 4 is a picture of an aquarium. The camera was placed so that the corner of the aquarium appears in the center of the picture.

\section{Figure 4}

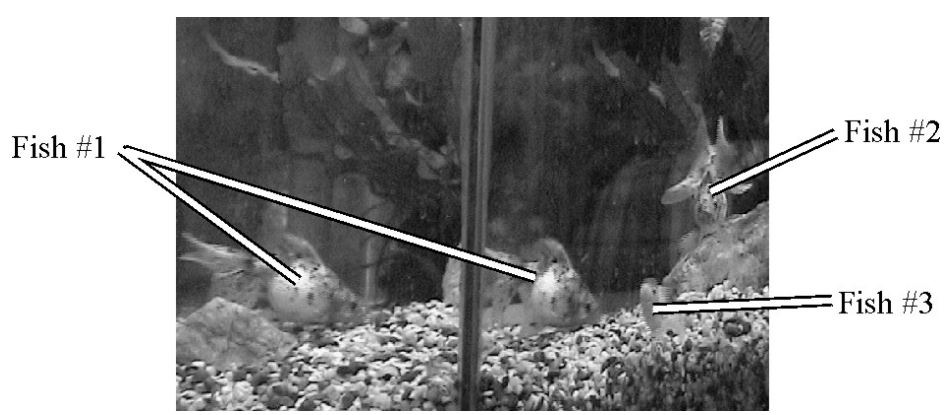

From this angle there appear to be four fish in the aquarium. In fact, there are only three. The two fish labeled 'Fish \#1' appear both to the left and to the right of the corner of the aquarium. For a person perceiving the aquarium from the same vantage point as the camera, real space contains four fish, even though the 


\section{Grammar and Gesture in American Sign Language}

aquarium only contains three. Not only does real space contain four fish, the locations of the real space fish do not conform to the locations of the actual physical fish. This is obvious since Fish \#1 appears to be in two different places. It does not even make sense to ask which one is the actual physical fish since neither one of the real space fish is in a location corresponding to the location of the physical fish.

In our daily lives, we move around and interact with our environment based on real space - our mental space representation of our environment. In general, the locations of the conceptual entities in real space correspond well to the locations of the physical things we interact with. The correspondence is so good, in fact, that we do not distinguish real space from reality. We operate as if real space were reality.

In mental space theory, meaning is constructed by making connections between entities within mental spaces. Fauconnier $(1985,1997)$ demonstrates that such mappings are a crucial, ongoing, and constant aspect of the use of any language. Thus, we would expect that mental space mappings also play a crucial role in constructing meaning in ASL.

The directionality of pronouns and indicating verbs is best understood as providing mental space mapping instructions. For example, suppose a signer is facing her addressee and directs the singular non-first person pronoun PRO toward a man to her right and to the left of the addressee. The addressee will recognize the sign PRO, which will lead the addressee to include the semantic structure of PRO ("one entity other than the signer") as part of the semantic structure of the utterance. Not only does the addressee recognize her production as an instance of PRO, the addressee also sees that PRO's directionality leads to the man to the left of the addressee.

Figure 5

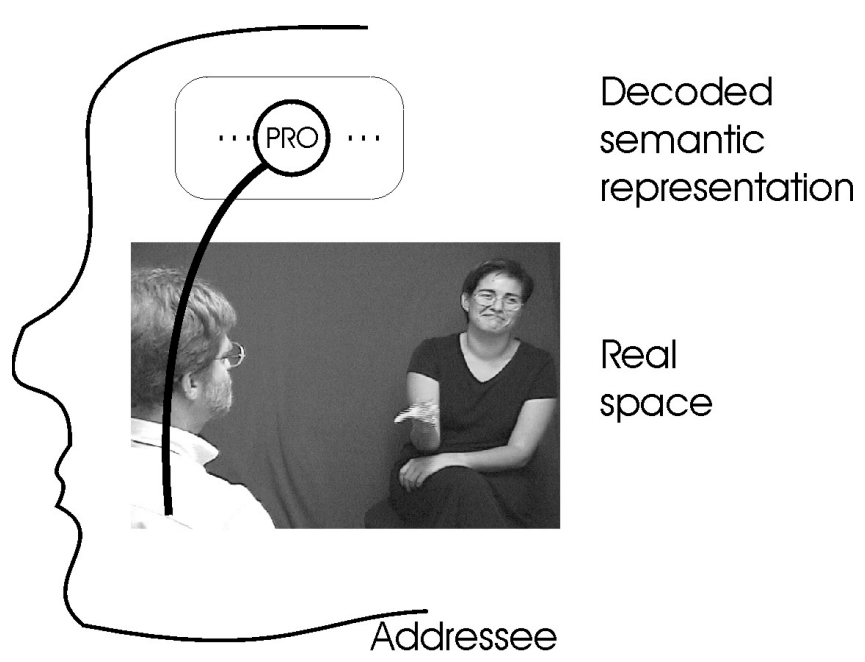

Figure 5 represents PRO as part of the semantic structure of the utterance being produced and also represents the addressee's real space. The significance of 
PRO's directionality is that it provides an instruction to make a mental space mapping between the semantic structure of PRO and the entity it is directed toward. Since PRO is directed toward the man to the left in the addressee's real space, PRO maps onto that man.

The directionality of indicating verbs is also best understood as providing mental space mapping instructions. In Figure 6a, for example, the sign ANALYZE is directed toward the addressee, directly ahead of the signer. In a cognitive grammar semantic representation, a verb like ANALYZE contains a trajector and a landmark. A trajector is the primary figure within a profiled relationship while the landmark is the secondary figure (Langacker 1987, 1991). In the active verb ANALYZE, the primary figure is the entity carrying out the analysis and the landmark is the entity being analyzed. In general, an indicating verb moves toward the entity to be mapped onto its landmark. ${ }^{2}$ Since ANALYZE is directed toward the addressee in real space, this prompts a mapping between him and the landmark, shown by the connector between the two in Figure $6 \mathrm{~b}$.

\section{Figure 6}

\section{a. ANALYZE}

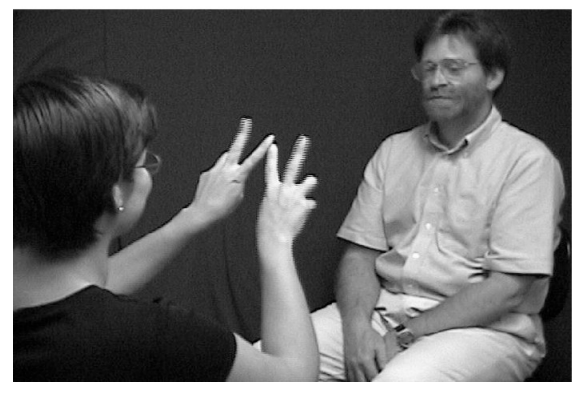

b. Mapped semantic structure

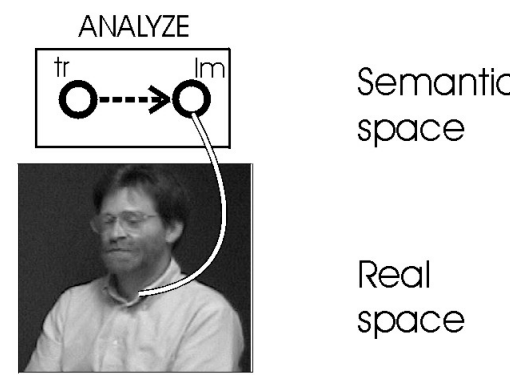

For verbs with a plural landmark, the directionality often takes the form of a sweeping motion that moves past the multiple entities, thereby indicating the extent of the group of entities. For example, Figure 7 illustrates two instances of COMPARE ${ }^{\text {[exhaustive] }}{ }^{3}$ In Figure 7 a, the hands move past a group of videotapes on a table in front of the signer. The sweeping movement of the hands indicates the entities to map onto the verb's landmark.

\footnotetext{
${ }^{2}$ There are some exceptional indicating verbs that move away from the entity to be mapped onto the landmark. Using a different analytical framework, Padden (1988) refers to them as "backwards verbs."

${ }^{3}$ Klima and Bellugi (1979) treat the sign shown in Figure 7a as a 'seriated external' inflection of the verb COMPARE. They analyze a vertically downward moving form of the sign as a 'seriated internal' inflection of COMPARE. In their analysis the difference in meaning between the two forms is inflectional. In the analysis I am describing in the text the difference in meaning between the two signs illustrated is not an inflectional difference but a referential one, based on the directionality of the sign.
} 
Another instance of COMPARE ${ }^{\text {[exhaustive] }}$ is illustrated in Figure $7 \mathrm{~b}$. In this case the verb makes a diagonally downward sweeping motion, indicating the diagonally arranged set of pictures.

\section{Figure 7}
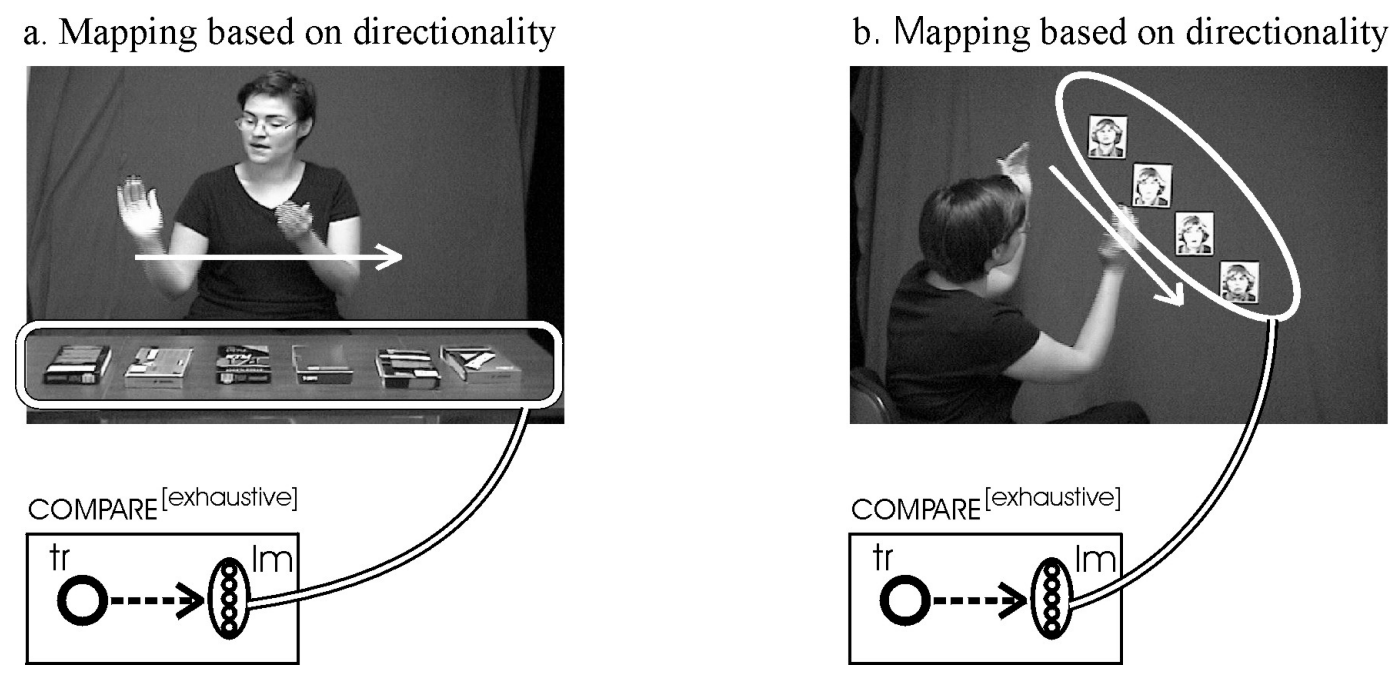

Figure $7 \mathrm{a}$ illustrates the mapping between the semantic structure of the verb and the set of videotapes indicated by the horizontal sweep of the hands. The mapping is motivated solely by the directional sweeping movement of the hands as the verb is produced. Figure $7 \mathrm{~b}$ shows the mapping that results from directing the same verb along a diagonal path past the pictures in Figure $7 \mathrm{~b}$. The same semantic structure maps onto a distinct set of entities.

\section{Mappings beyond the Individual Sign}

Thus far I have restricted the discussion of directionality to individual signs. I have attempted to demonstrate that the directionality of both pronouns and indicating verbs is best understood as identifying one or more entities to be mapped onto the semantic structure of the sign itself. Next I will describe what happens when a verb and its pronominal subject both point.

\section{Figure 8}

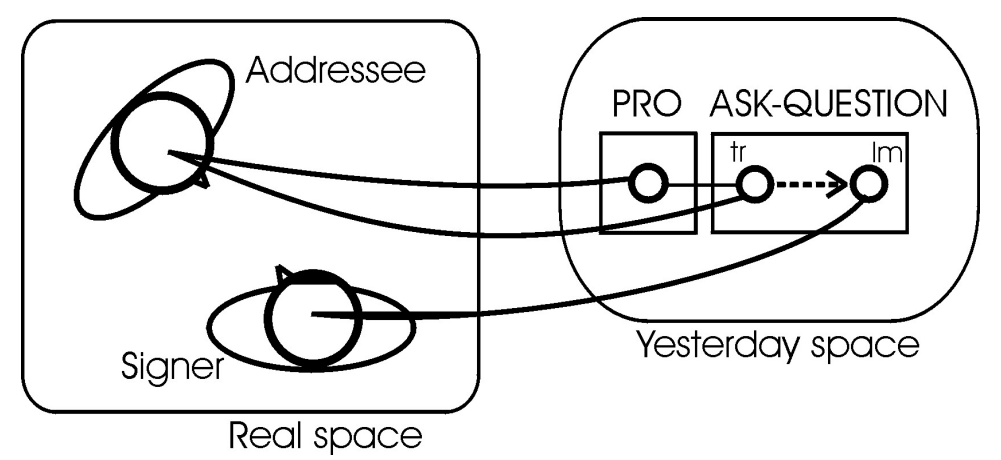


Figure 8 illustrates the mapped semantic structure of the clause PRO ASKQUESTION YESTERDAY. In this instance, the subject, PRO, is directed toward the addressee. The verb ASK-QUESTION begins its movement directed toward the addressee then moves in a straight path toward the signer.

Following Langacker (1999), I treat semantic structures as mental spaces. Thus, I am representing the semantic structure of the three-sign clause PRO ASKQUESTION YESTERDAY as a mental space with the setting 'yesterday'. Because of the subject-verb relationship between PRO and ASK-QUESTION, the semantic structure of the pronoun and the verb's trajector are linked. The horizontal connector between those two conceptual entities represents this connection.

Directing the subject pronoun PRO toward the addressee is an instruction to map the real space addressee onto the entity profiled in the semantic structure of the pronoun. Beginning the verb ASK-QUESTION toward the addressee is an instruction to map the addressee onto its trajector. Moving the verb along a path toward the signer is an instruction to map the landmark onto the signer. The resulting set of mappings involves two mental spaces: the semantic representation of the clause and real space. Simply following the mapping instructions from the directionality of the individual signs creates a coherent representation of the constructed meaning of this utterance.

This example is typical in that the directionality of a subject pronoun and the verb provide mapping instructions that result in the subject pronoun and the trajector being mapped onto the same entity - in this case, the addressee in real space. This does not always happen, however, as will become apparent later.

\section{Making Entities Present through Blending}

In the next example the signer is discussing the number of minutes in college and professional basketball games. He begins with the sign BASKETBALL, marked as a topic by raising the eyebrows and tilting his head back (Liddell 1980).

Next he makes college and professional basketball present in the space ahead of him by producing the sign COLLEGE to the left of center and \#PRO ('professional') to the right of center, as illustrated in the second and third frames of Figure 9. The entities 'college basketball' and 'professional basketball' are quite abstract. Nevertheless, mental space blending (Fauconnier and Turner 1996, Fauconnier 1997) allows the signer to make those abstract entities present.

Next he directs one instance of PRO toward the area of space blended with professional basketball, which I label |professional basketball|, and directs a second instance of PRO toward the area of space labeled |college basketball|. The two instances of PRO serve as the dual subject of the side-to-side moving verb SAME. This particular instance of SAME moves back and forth between |college basketball| and |professional basketball|.

The directionality of the two pronouns and the verb in this example work just like the previous example where the actual referents were physically present. In this case, the abstract entities 'college basketball' and 'professional basketball' were made to be present through blending with the space ahead of the signer. 


\section{Grammar and Gesture in American Sign Language}

Since blending made them present, both of the pronouns and the verb can be directed toward them. The mappings associated with the final three signs in Figure 9 are illustrated in Figure 10.

Figure 9 "As for basketball, are college and professional games the same (with respect to their duration)."
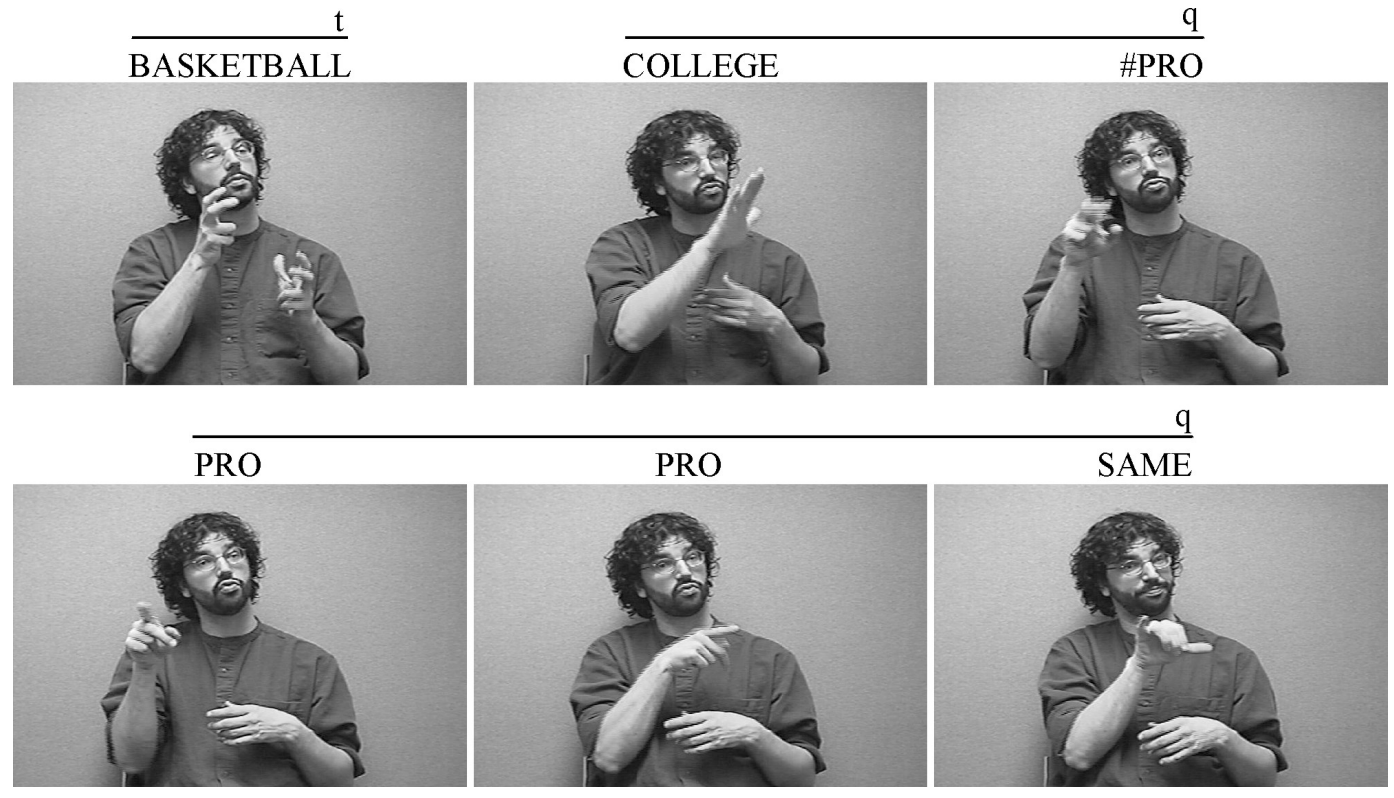

Figure 10

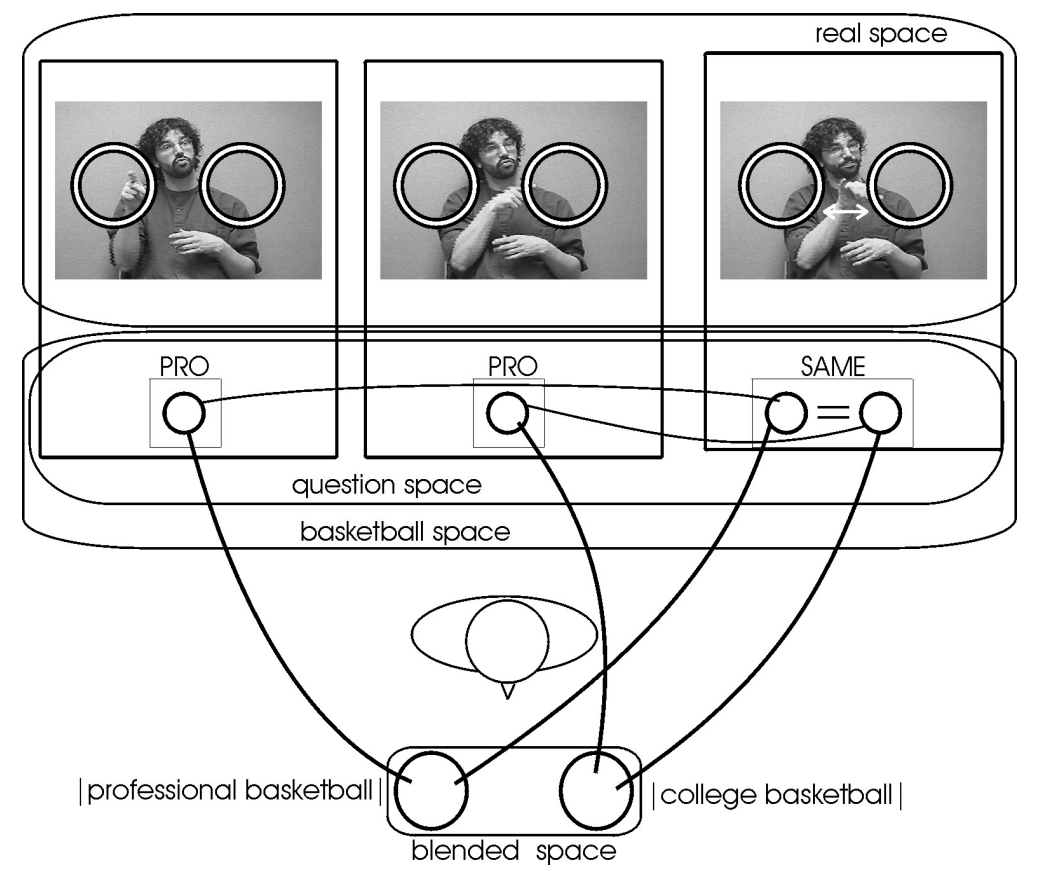


The semantic structure of the first instance of PRO is mapped onto |professional basketball| because the sign is directed toward it. The second instance of PRO is directed toward and therefore mapped onto |college basketball|. Finally, the two conceptual entities within the semantic structure of SAME are mapped onto the two blended entities ahead of the signer, also because the sideto-side motion of SAME points at both entities.

The next example is especially interesting because the subject pronoun maps onto the addressee but the verb's trajector does not. The signer is asking the addressee why he was staring at the side of her head yesterday.

YESTERDAY PRO LOOK-AT ${ }^{\text {[durational] }}$. WHAT'S-UP.

"Yesterday you were staring at the side of my head. What was that about?"

In (1) PRO points at the physically present addressee. The verb LOOK$\mathrm{AT}^{\text {[durational] }}$ points at two other entities. The fingertips point in the direction of looking - the side of the signer's head - while the back of the hand points at the entity doing the looking. But the back of the hand is not directed toward the addressee. Instead, the back of the hand is pointing to the right of the signer. The directionality of PRO would seem to indicate that the addressee carried out the action while the directionality of the verb indicates that an entity to the right of the signer carried out the action.

Figure 11

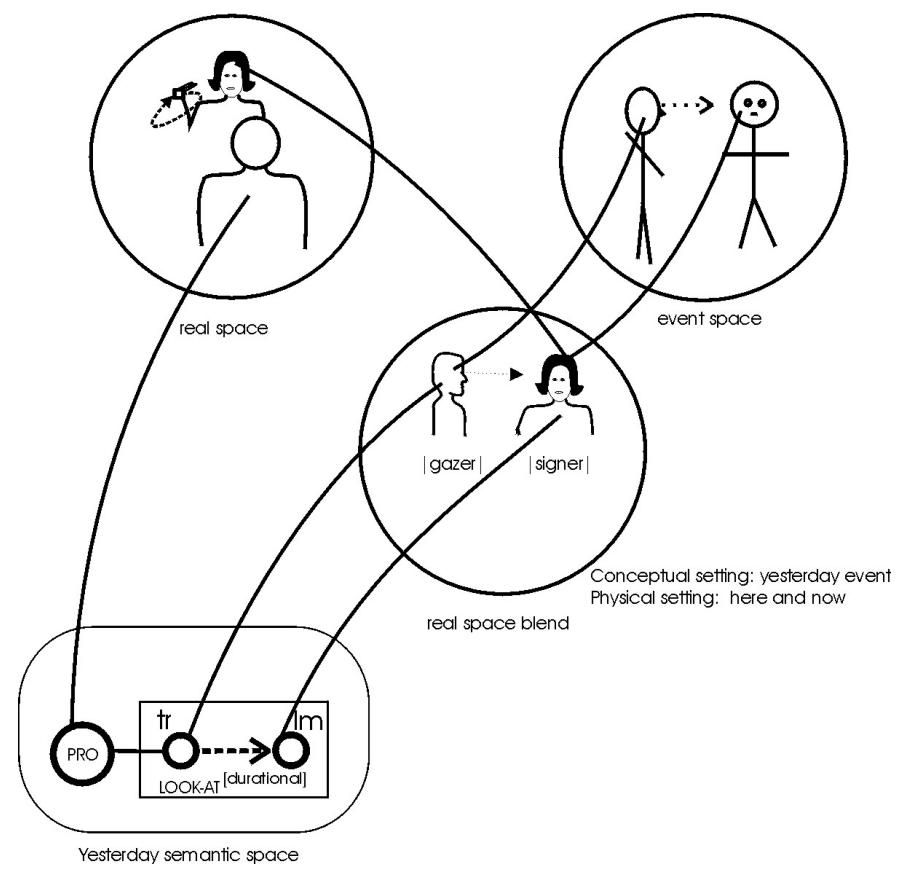




\section{Grammar and Gesture in American Sign Language}

In order to understand this example, it is necessary to describe the complex conceptualizations that underlie its use. First, the signer is describing an event that took place yesterday, represented as the 'event space' in the upper right in Figure 11. The sign LOOK-AT ${ }^{\text {[durational] }}$ is being produced in real space, upper left in Figure 11. Through mental space blending, she projects aspects of the event space onto real space. The signer projects herself from the 'event space' onto herself in real space. I have labeled the resulting blended entity as the |signer|. ${ }^{4}$ The person staring at her is also projected to the right of the |signer| in the blended space. I have labeled this entity as the |gazer|.

Through blending, the signer has recreated the scene in which the current addressee was staring at the side of her head yesterday. Only part of the scene is visible. The addressee can see the |signer| but cannot see the |gazer|. Because of the existence of the real space blend, there are two 'realities' that signs can be directed toward. One is real space and the other is the real space blend. An additional characteristic of the blended space adds to the complexity of this example. The blended space exists in the same place as real space. A non-signing passerby only has access to real space. The addressee, however, not only has access to real space, but also understands that aspects of the event that took place yesterday are also visible in the blend. Another interesting aspect of the blended space is that it has two distinct settings. It inherits its conceptual setting from the 'yesterday' event space. It inherits its physical setting from real space. Thus, it is a here-and-now partial recreation of the event that took place yesterday.

Given the existence of these two overlapping spaces, we can now explore the function of the pointing seen in both the subject and the verb. I will begin with the verb. During the production of LOOK-AT ${ }^{\text {[durational] }}$ the back of the hand indicates that the |gazer| was doing the looking. The directionality of the fingertips indicates that the looking was directed toward the side of the head of the |signer|. If we make the assumption that the subject of the verb identifies the entity doing the looking, then the pointing of the subject pronoun PRO indicates that the current addressee was the one doing the looking. The result is a conceptually coherent set of mappings. The verb's directionality identifies the two participants in the real space blend. The verb's directionality does not, however, provide the identity of the |gazer|. The identity of the |gazer| only becomes apparent by directing the subject PRO toward the real space addressee.

\section{Implications for Meaning Construction}

Currently, meaning construction is conceived of as taking place at a level distinct from language structure:

\footnotetext{
${ }^{4}$ The signer only partially becomes herself yesterday. That is, it is primarily her head that represents herself yesterday. The signer is still using her right hand in real space to narrate.
} 
Scott K. Liddell

Meaning construction takes place at a "cognitive" level, call it level $C$; this level is distinct from the language structure (i.e. it is not an "underlying form," it is not a "representation" of language or of language meaning, it is not objectively associated with any particular set of linguistic expressions). (Fauconnier 1997:36)

I have not been treating meaning construction as something that takes place at a level distinct from language structure here since the directional ASL signs appear to require mappings between their individual semantic structures and other mental space entities. Although it might be possible that sign languages and vocally produced languages operate differently with respect to meaning construction, it could also be possible that constructing meaning by mapping semantically encoded meaning onto other mental space elements can adequately account for meaning construction in both signed languages and vocally produced languages.

It is not possible to provide a thorough account here of how this approach to meaning construction can account for the wide array of data for which mental space theory has already demonstrated revealing solutions. However, below I will describe how this approach can account for the role-value distinction. I will then suggest an approach to metonymy, which also differs from the current mental space approach to metonymy.

I will use the sentence Bob's neighbor bought an island to illustrate the distinction between roles and values.

\section{Figure 12}

a.

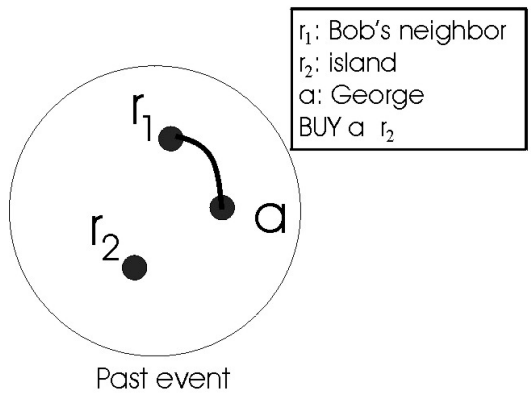

b.

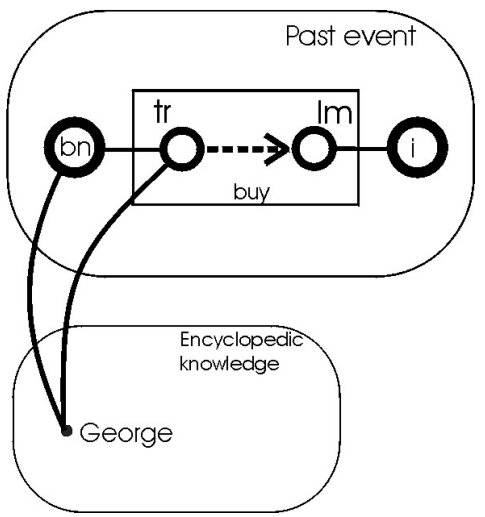

Figure 12a contains a mental space structured according the proposals in Fauconnier (1997). Entities $r_{1}$ and $r_{2}$ represent the two roles 'Bob's neighbor' and 'an island'. Assuming that the addressee knows that the speaker is talking about his neighbor George, entity $\mathrm{r}_{1}$ is mapped onto entity $a$ (George). Entity $\mathrm{r}_{2}$ is unmapped and remains a role, unmapped onto a specific value.

In Figure 12b Bob's neighbor maps onto the entity George in the addressee's encyclopedic knowledge. Since there is no metonymy in this example, the trajector likewise maps onto George. However, the addressee does not know which 


\section{Grammar and Gesture in American Sign Language}

island is being talked about. In this circumstance, the semantic structure of an island remains unmapped.

A comparison of the two diagrams shows that the role $\mathrm{r}_{1}$ in Figure 12a corresponds to the semantic structure of Bob's neighbor in Figure 12b. Similarly, $\mathrm{r}_{2}$ corresponds to the semantic structure of an island. A conceptual role is an entity with a specific semantic property. That is, the role 'an island' is a conceptual entity with the property 'island'. This is exactly how one would describe the semantic structure of the noun phrase an island. As a result, the semantic poles of the subject and object noun phrases above could be described as expressing roles. Since Bob's neighbor maps onto George, the subject NP has a value reading. Thus, without the need to propose role-type entities, semantic representations automatically provide roles. When mapped onto other mental space entities, they express value readings.

Metonymy describes the use of language where a speaker mentions one thing in order talk about another. Fauconnier (1985) proposes a solution to metonymy in which, given the appropriate pragmatic circumstances, mentioning one entity $a$ can lead directly to a related entity $b$. Both $a$ and $b$ are mental space elements linked by a connector. Element $a$ is called the trigger and element $b$ is called the target. The ambiguous example In that picture, the girl with green eyes has blue eyes can be understood to mean that the image has green eyes while the model has blue eyes, or vice versa. ${ }^{5}$

\section{Figure 13}

a

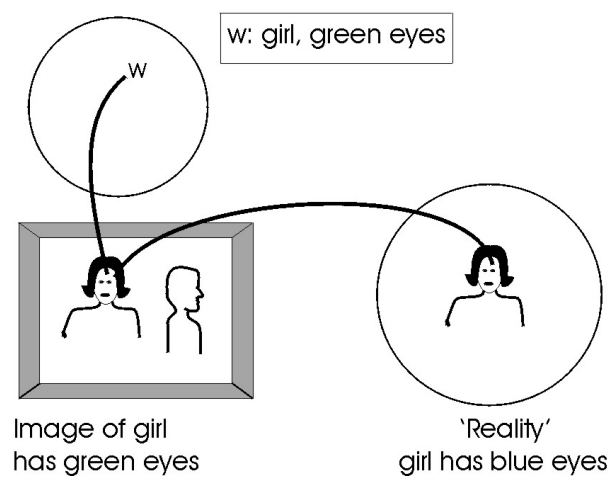

b.

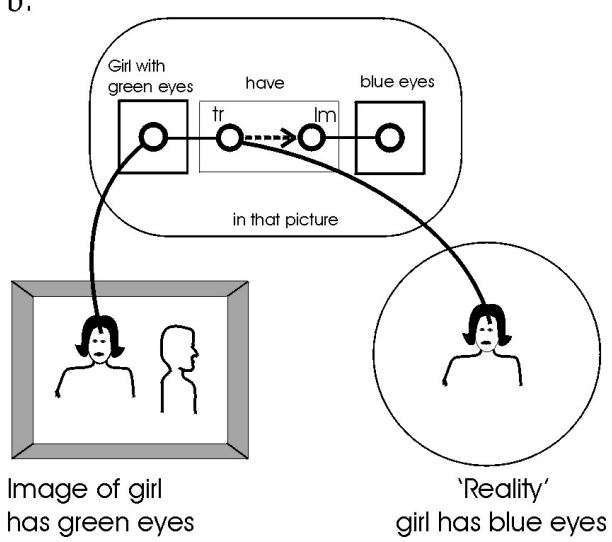

The mapping shown in Figure 13a illustrates the constructed meaning in which the image of the girl has green eyes while the model has blue eyes. Entity $w$ maps onto the image of the girl (the trigger), which does have green eyes. The image maps onto the model (the target), who has blue eyes.

If semantic spaces of the type proposed by Langacker $(1987,1991)$ are used for meaning construction, an alternative suggests itself. The directionality of ASL

\footnotetext{
5 This example is similar to an example from Fauconnier (1985).
} 
Scott K. Liddell

pronouns and verbs has already demonstrated that overt subjects and trajectors can be independently mapped in the process of meaning construction. In metonymy a person mentions one thing in order to talk about another. The solution proposed here is a direct representation of that description of metonymy. The subject the girl with green eyes maps in a straightforward way onto the image of a girl with green eyes. If the semantic structure and the mapping of an overt subject is taken as a guide in determining the mapping of the trajector, then the addressee is free to conclude that the entity with blue eyes is, in fact, the model. This allows a mapping between the trajector and the girl with blue eyes. Subjects and trajectors will typically map onto the same entity, but not in the case of metonymy, where the speaker mentions one thing in order to talk about another.

\section{Conclusion}

The directionality of pronouns and verbs in ASL provides mapping instructions for their semantic structures. This leads to a type of meaning construction in which the mental spaces that serve as the basis for meaning construction are semantic structures. If such mappings are generalized to other instances of language use, both spoken and signed, it appears that the benefits of mental space mappings are maintained. Semantic structures are an indispensable component of the chain linking phonetics to comprehension. The data discussed in this paper suggest that meaning can be satisfactorily constructed if built around these obligatory representations.

\section{References}

Edge, VickiLee, and Leora Herrmann. 1977. Verbs and the Determination of Subject in American Sign Language. In L. Friedman (ed.), On the Other Hand. New York: Academic Press, 137-179.

Fauconnier, Gilles. 1985. Mental Spaces. Cambridge, MA: MIT Press. Reprinted 1994, Cambridge, UK: Cambridge University Press.

Fauconnier, Gilles. 1997. Mappings in Thought and Language. Cambridge, UK: Cambridge University Press.

Fauconnier, Gilles, and Mark Turner. 1996. Blending as a Central Process of Grammar. In Adele Goldberg (ed.), Conceptual Structure, Discourse and Language. Stanford, CA: CSLI Publications, 113-130.

Fischer, Susan. 1975. Influences on Word Order Change in ASL. In C. N. Li (ed.), Word Order and Word Order Change. Austin, TX: University of Texas Press, $1-25$.

Friedman, Lynn. 1975. Space, Time, and Person Reference in American Sign Language. Language 51:940-961.

Gee, James, and Judy Kegl. 1982. Semantic Perspicuity and the Locative Hypothesis: Implications for Acquisition. Journal of Education 164(2). 
Klima, Edward S., and Ursula Bellugi. 1979. The Signs of Language. With R. Battison, P. Boyes Braem, S. Fischer, N. Frishberg, H. Lane, E. M. Lentz, D. Newkirk, E. Newport, C. C. Pedersen, and P. Siple. Cambridge, MA: Harvard University Press.

Lacy, Richard. 1974. Putting Some of the Syntax back into Semantics. Ms., The Salk Institute.

Langacker, Ronald W. 1987. Foundations of Cognitive Grammar, Vol. I: Theoretical Prerequisites. Stanford, CA: Stanford University Press.

Langacker, Ronald W. 1991. Foundations of Cognitive Grammar, Vol. II: Descriptive Application. Stanford, CA: Stanford University Press.

Langacker, Ronald W. 1999. Discourse in Cognitive Grammar. Paper presented at the International Cognitive Linguistics Association. Stockholm, Sweden, July 1999.

Liddell, Scott K. 1980. American Sign Language Syntax. The Hague: Mouton.

Liddell, Scott K. 1994. Tokens and Surrogates. In Inger Ahlgren, Brita Bergman, and Mary Brennan (eds.), Perspectives on Sign Language Structure: Papers from the Fifth International Symposium on Sign Language Research, Vol. I. University of Durham, England: The International Sign Linguistics Association, 105-119.

Liddell, Scott K. 1995. Real, Surrogate, and Token Space: Grammatical Consequences in ASL. In Karen Emmorey and Judy Reilly (eds.), Language, Gesture, and Space. Hillsdale, NJ: Lawrence Erlbaum Associates Publishers, 1941.

Neidle, Carol, Dawn MacLaughlin, Judy Kegl, Ben Bahan, and Debra Aarons. 1995. Overt Realization of Syntactic Features in American Sign Language. Syntax seminar, University of Trondheim, Norway, May 1995.

Padden, Carol. 1988. Interaction of Morphology and Syntax in American Sign Language. New York: Garland Publishing.

Stokoe, William C. 1960. Sign Language Structure: An Outline of the Visual Communication System of the American Deaf. Studies in Linguistics Occasional Papers, No. 8.

Woodward, James. 1970. Personal Pronominalization in American Sign Language. Ms., Georgetown University.

Department of Linguistics

Gallaudet University

Dawes House

800 Florida Avenue, NE

Washington, DC 20002

scott.liddell@gallaudet.edu 